\title{
AXISYMMETRIC STAGNATION FLOW ON A CYLINDER*
}

\author{
By CHANG-YI WANG (Michigan State University)
}

Due to the inherent nonlinearity of the Navier-Stokes equations, there exist only three exact solutions of stagnation flows: Hiemenz [1] found a solution to the twodimensional stagnation flow against a plate, Homann [2] investigated the axisymmetric stagnation flow, also against a plate, and Howarth [3] and Davey [4] extended the results to unsymmetric cases.

The present note presents a new exact solution, namely, axisymmetric stagnation flow on an infinite circular cylinder. Fig. 1 shows a cylinder described by $r=a$ in the cylindrical polar coordinates. The flow is axisymmetric about the $z$ axis and also symmetric to the $z=0$ plane. The stagnation "line" is at $z=0, r=a$. This flow may be useful in certain cooling processes.

Let $u$ and $w$ be the velocities in the directions $r$ and $z$ respectively. If the flow is inviscid, the potential velocity and pressure distribution in the neighborhood of the stagnation line are

$$
\begin{aligned}
u & =-k\left(r-a^{2} / r\right), \\
w & =2 k z, \\
p & =p_{0}-\rho k^{2}\left[2 z^{2}+\frac{1}{2}\left(r-a^{2} / r\right)^{2}\right],
\end{aligned}
$$

where $k$ is a given constant of dimensions [1/T], $p_{0}$ is the stagnation pressure, and $\rho$ is the density. We expect the viscous flow to approach the potential solution as $r \rightarrow \infty$.

The constant-density Navier-Stokes equations in cylindrical coordinates are

$$
\begin{gathered}
u u_{r}+w u_{z}=-\frac{1}{\rho} p_{r}+\nu\left(u_{r r}+\frac{1}{r} u_{r}+u_{z z}-\frac{u}{r^{2}}\right) \\
u w_{r}+w w_{z}=-\frac{1}{\rho} p_{z}+\nu\left(w_{r r}+\frac{1}{r} w_{r}+w_{z z}\right), \\
r w_{z}+(r u)_{r}=0 .
\end{gathered}
$$

Let

$$
\begin{aligned}
u & =-k a \eta^{-1 / 2} f(\eta), \\
w & =2 k f^{\prime}(\eta) z,
\end{aligned}
$$

where $\eta=(r / a)^{2}$. After some algebra, Eqs. (4) and (5) reduce to

$$
\begin{aligned}
& \eta f^{\prime \prime \prime}+f^{\prime \prime}+R\left(1+f f^{\prime \prime}-f^{\prime 2}\right)=0, \\
& p=p_{0}-\rho\left(\frac{k^{2} a^{2}}{2} \frac{f^{2}}{\eta}+2 v k f^{\prime}+2 k^{2} z^{2}\right),
\end{aligned}
$$

* Received February 9, 1973; revised version received May 20, 1973. 
where $R \equiv k a^{2} / 2 \nu$ is a Reynolds number. The boundary conditions are

$$
f(1)=0, \quad f^{\prime}(1)=0, \quad f^{\prime}(\infty)=1 .
$$

Eq. (9) is integrated numerically by the Runge-Kutta method. The accuracy is determined by varying the step size. Figs. 2 and 3 shows $f(\eta)$ and $f^{\prime}(\eta)$ respectively for several values of $R$. Due to the natural length scale $a$ which enters in the parameter

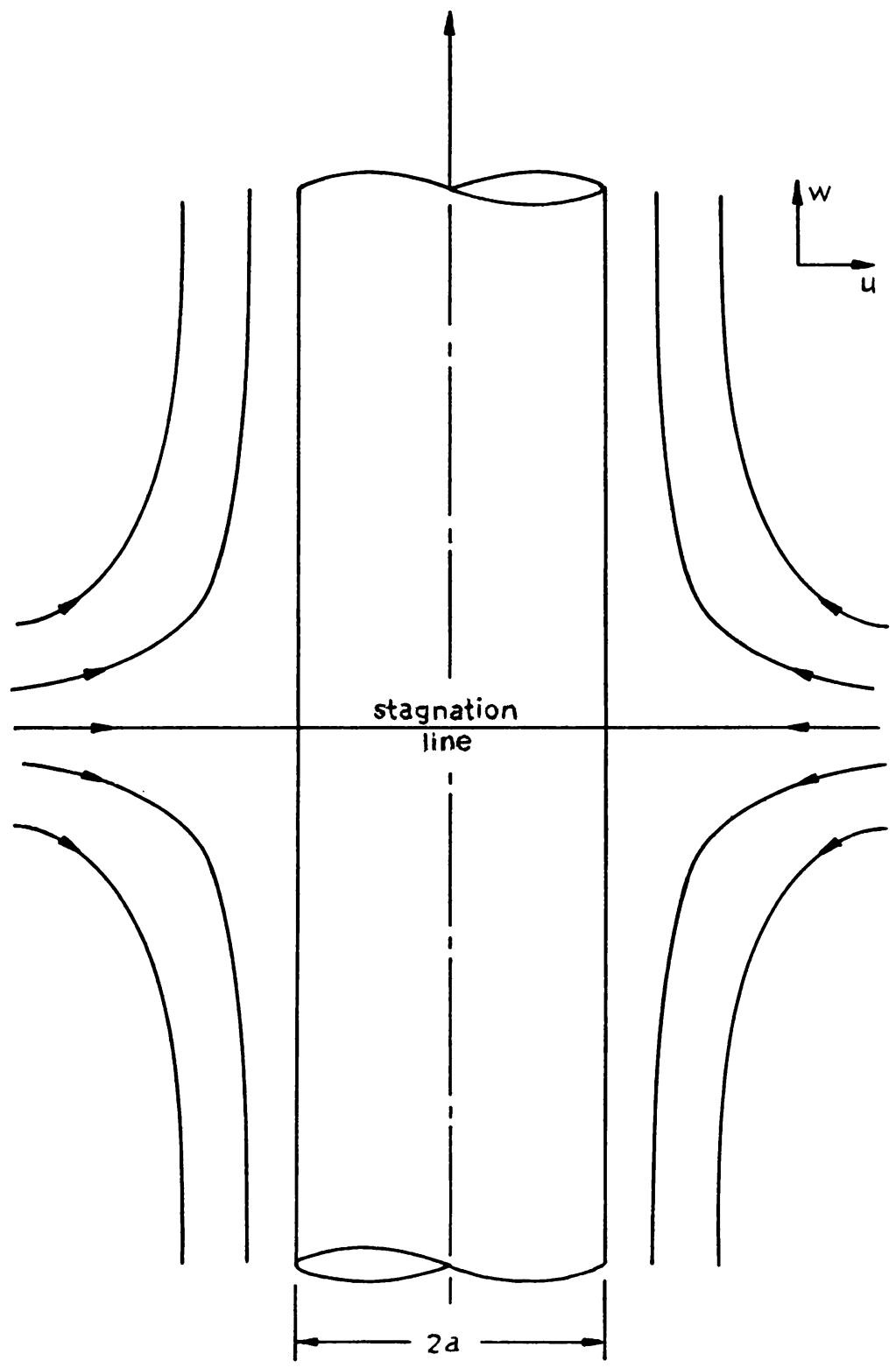

Fig. 1. The coordinate axis. 
NOTES

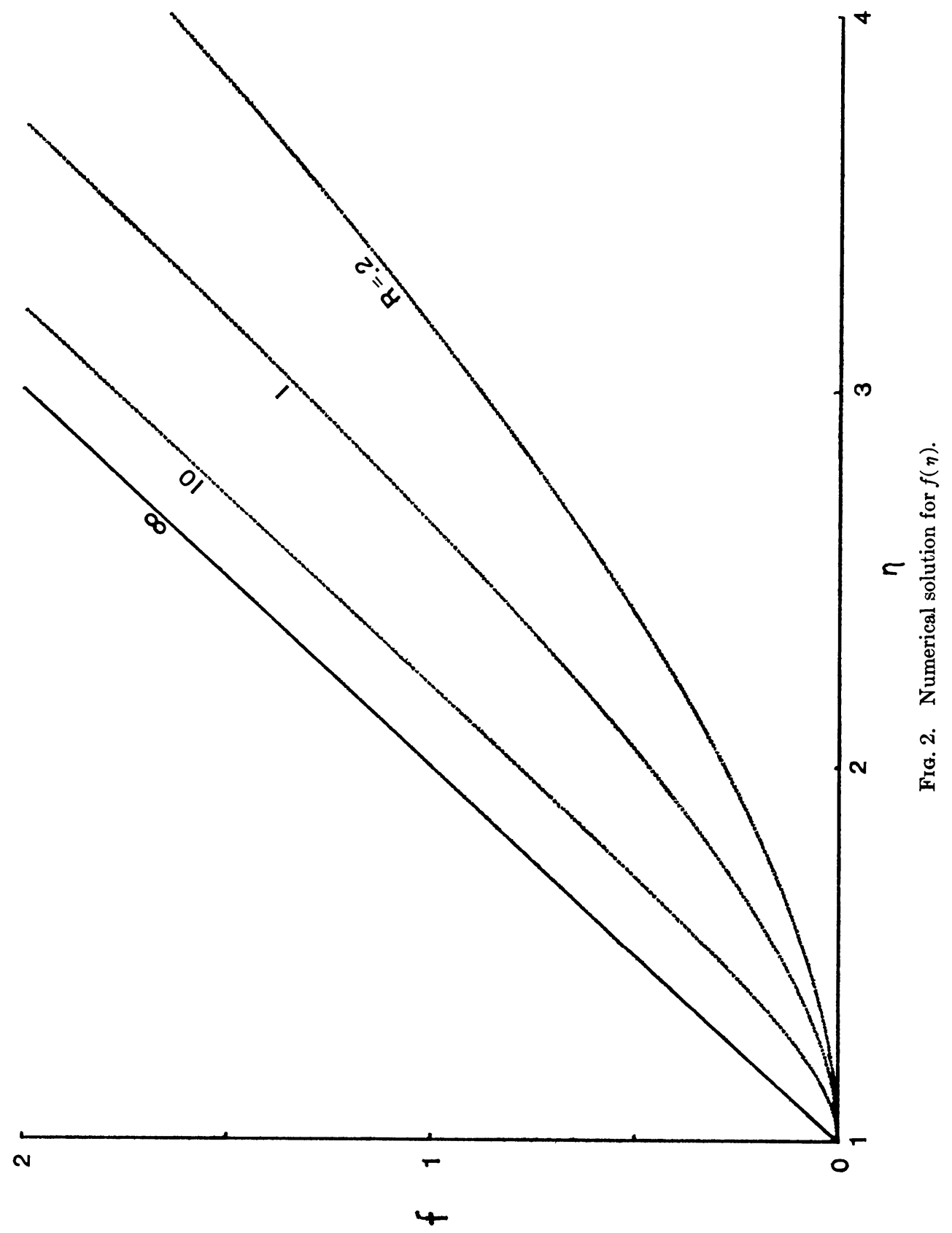

$R$, these curves are not similar to each other. The numerical results are given in Tables I, II, III.

Of some interest is the asymptotic behavior for large $\eta$. Let $f(\eta)=\eta+c+g(\eta)$ where $c$ is a constant and $g(\eta)$ is small. Then Eq. (9) linearizes to

$$
\xi g^{\prime \prime \prime}(\xi)+(1+\xi) g^{\prime \prime}-2 g^{\prime}=0
$$


210

CHANG-YI WANG

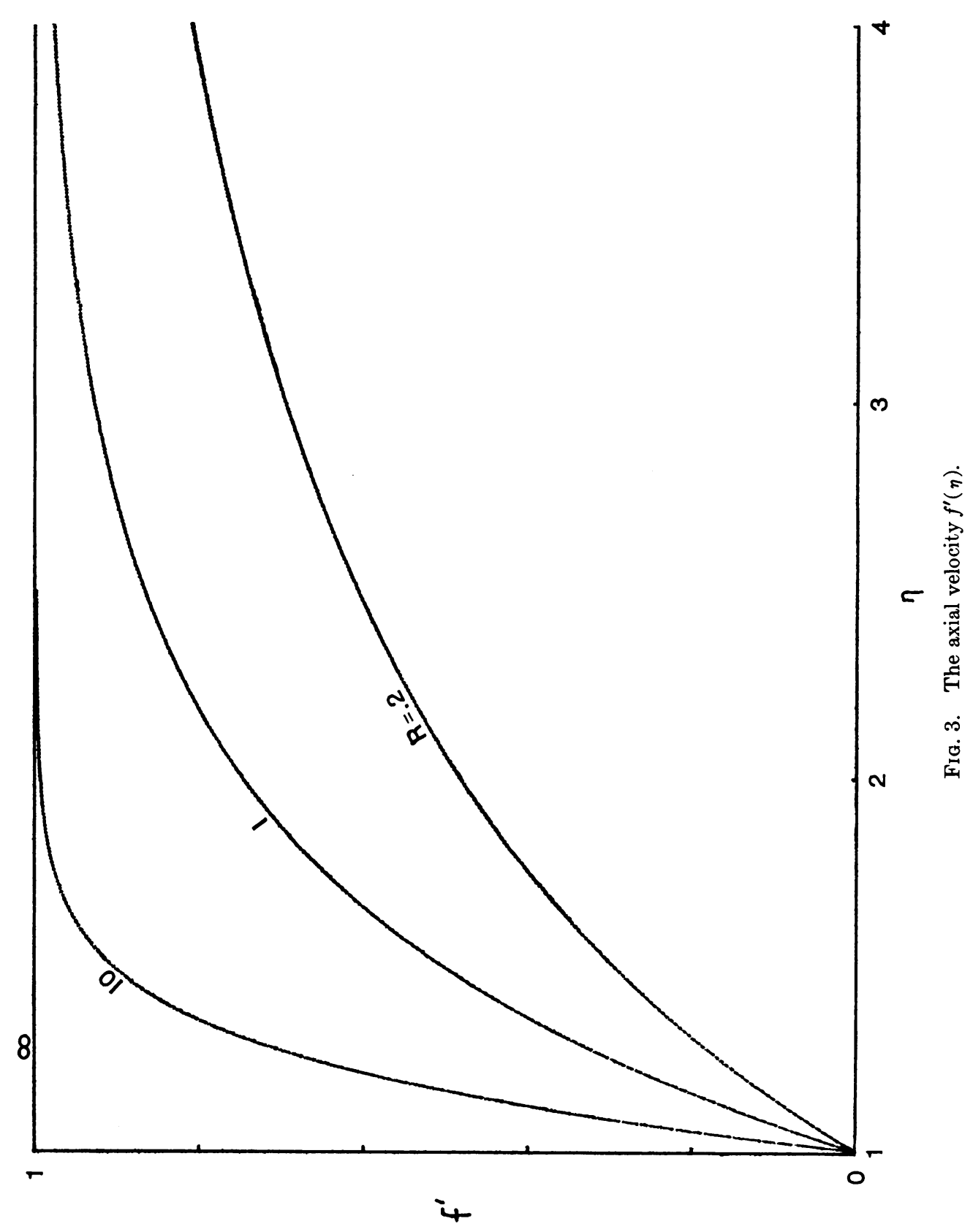

where $\xi=R \eta$. This shows the solutions are at least similar for large $\eta$. The nontrivial solution which decays to zero at infinity is found to be

$$
g(\xi)=\text { const } \int_{\infty}^{\xi}\left[\left(\lambda^{2}+4 \lambda+2\right) \int_{\infty}^{\lambda} \beta^{-1}\left(\beta^{2}+4 \beta+2\right)^{-2} e^{-\beta} d \beta\right] d \lambda .
$$

Eq. (13) can be rewritten in terms of one integral 


$$
g(\xi)=\text { const } \int_{\infty}^{\xi}\left(\frac{\xi^{3}}{3}+2 \xi^{2}+2 \xi-\frac{t^{3}}{3}-2 t^{2}-2 t\right) t^{-1}\left(t^{2}+4 t+2\right)^{-2} e^{-t} d t .
$$

The decay is proportional to $\xi^{-3} e^{-\xi}$.

For large $R$ the solution is closely related to the two-dimensional stagnation flow against a flat plate. The transformation

$$
f(\eta)=R^{1 / 2} \varphi(\xi)
$$

where $\xi=R^{1 / 2}(\eta-1)$ yields Hiemenz's equation

$$
\begin{gathered}
\varphi^{\prime \prime \prime}+\varphi \varphi^{\prime \prime}-\varphi^{\prime 2}+1=0, \\
\varphi(0)=\varphi^{\prime}(0)=0, \quad \varphi^{\prime}(\infty)=1
\end{gathered}
$$

as a first approximation. The error is of order $R^{-1 / 2}$. Table IV gives a comparison to Hiemenz's value.

If the Reynolds number were small, Eq. (9) yields as first approximation

$$
f=\text { const }(\eta \ln \eta-\eta+1)
$$

which is singular at infinity. The solution breaks down at a distance of $\eta=0(1 / R)$,

$$
\text { TABLE I } \quad(\mathrm{R}=.2)
$$

\begin{tabular}{rrrr}
$\eta$ & \multicolumn{1}{c}{ f } & \multicolumn{1}{c}{$\mathbf{f}^{\prime}$} & $\mathbf{f}^{\prime \prime}$ \\
1.0 & & 0 & .78605 \\
1.5 & .08170 & .29998 & .45864 \\
2.0 & .28134 & .48534 & .29900 \\
2.5 & .55708 & .61005 & .20737 \\
3.0 & .88537 & .69829 & .14970 \\
3.5 & 1.2514 & .76290 & .11117 \\
4.0 & 1.6456 & .81139 & .08432 \\
4.5 & 2.0609 & .84848 & .06503 \\
5.0 & 2.4926 & .87726 & .05083 \\
6.0 & 3.3917 & .91786 & .03205 \\
7.0 & 4.3235 & .94386 & .02087 \\
8.0 & 5.2766 & .96100 & .01392 \\
9.0 & 6.2437 & .97254 & .00947 \\
10.0 & 7.2204 & .98045 & .00654 \\
12.0 & 9.1918 & .98982 & .00324 \\
14.0 & 11.176 & .99455 & .00167 \\
16.0 & 13.168 & .99702 & .00088 \\
18.0 & 15.164 & .99834 & .00048 \\
20.0 & 17.161 & .99906 & .00026 \\
25.0 & 22.159 & .99977 & .00006 \\
30.0 & 27.158 & .99995 & .00001 \\
35.0 & 32.158 & 1.00000 & .00000
\end{tabular}


TABLE II $(\mathrm{R}=1$.

\begin{tabular}{cccc}
$\eta$ & \multicolumn{1}{c}{ f } & $f^{\prime}$ & $f^{\prime \prime}$ \\
1 & & 0 & 1.484185 \\
1.2 & .02667 & .25302 & 1.07223 \\
1.4 & .09665 & .43724 & .78662 \\
1.6 & .19836 & .57315 & .58369 \\
1.8 & .32361 & .67444 & .43697 \\
2.0 & .46647 & .75054 & .32949 \\
2.5 & .87488 & .86968 & .16664 \\
3.0 & 1.3266 & .93068 & .08647 \\
3.5 & 1.8008 & .96261 & .04572 \\
4.0 & 2.2867 & .97961 & .02453 \\
4.5 & 2.7791 & .98878 & .01331 \\
5.0 & 3.2748 & .99378 & .00729 \\
6.0 & 4.2712 & .99805 & .00224 \\
7.0 & 5.2700 & .99938 & .00070 \\
8.0 & 6.2697 & .99980 & .00022 \\
9.0 & 7.2695 & .99993 & .00007 \\
10.0 & 8.2695 & .99998 & .00002 \\
11.0 & 9.2695 & 1.00000 & .00000
\end{tabular}

TABLE III $(\mathrm{R}=10$.

$\eta$

f

$\mathrm{f}^{\prime}$

$\mathrm{f}^{\prime \prime}$

1.0

0

0

.35045

.58982

.74753

.84821

.91071

1.5

1.6

1.7

1.8

1.9

2.0

2.2

2.4

.01857

.06638

.13382

.21400

.30220

.39532

.49139

.58919

.68797

.78731

.98677

2.6

2.8

1.1866

1. 3865

3.0

1.5865

3.5

1. 7865

2. 2865

.94852

.97087

.98380

.99114

.99522

.99867

.99965

.99991

.99998

.99999

4.16292

2. 89754

1. 93962

1. 25571

.78923

.48298

.28845

.16847

.09640

.05412

.02986

.00866

.00238

.00062

.00015

.00004

1.00000

.00000 


\section{TABLE IV}

$\begin{array}{rlr}R & R^{-1 / 2} f^{\prime \prime}(1) \\ .2 & 1.7577 & \\ 1 . & 1.484185 & \\ 10 . & 1.31643 \\ \infty & 1.232588 \quad \text { (Hiemenz flow, } \varphi^{\prime \prime}(0) \text { ) }\end{array}$

where, in order to bring in the nonlinear terms, we set $f=(1 / R) h(\xi), \xi=R \eta$. One obtains

$$
\xi h^{\prime \prime \prime}+h^{\prime \prime}+1+h h^{\prime \prime}-h^{\prime 2}=0
$$

which is an equation as difficult as the original one. As $\eta$ is increased further, linearization is possible and we obtain Eq. (12) by setting $h(\xi)=\xi+c+g(\xi)$.

The author is indebted to a referee for his comments on the last two paragraphs.

\section{REFERENCES}

[1] K. Hiemenz, Die Grenzschicht an einem in den gleichförmingen Flüssigkeitsstrom eingetauchten geraden Kreiszylinder, Dinglers J. 326, 321-410 (1911)

[2] F. Homann, Der Einfluss grosser Zähigkeit bei der Strömung um den Zylinder und um die Kugel, Z. angew. Math. Mech. 16, 153-164 (1936)

[3] L. Howarth, The boundary layer in three-dimensional flow. Part II: The flow near a stagnation point, Phil. Mag. (7) 42, 1433-1440 (1951)

[4] A. Davey, Boundary-layer flow at a saddle point of attachment, J. Fluid Mech. 10, 593-610 (1961) 